\title{
Multi-notch holographic filters for atmospheric lines suppression
}

Sebastien Blais-Ouellette, Etienne Artigau, Frank Havermeyer, Keith Matthews, Christophe Moser, et al.

Sebastien Blais-Ouellette, Etienne Artigau, Frank Havermeyer, Keith Matthews, Christophe Moser, Demetri Psaltis, Gregory J. Steckman, "Multinotch holographic filters for atmospheric lines suppression," Proc. SPIE 5494, Optical Fabrication, Metrology, and Material Advancements for Telescopes, (24 September 2004); doi: 10.1117/12.552116

Event: SPIE Astronomical Telescopes + Instrumentation, 2004, Glasgow, United Kingdom 


\title{
Multi-Notch Holographic Filters for Atmospheric Lines Suppression
}

\author{
Sébastien Blais-Ouellette ${ }^{a, c}$, Étienne Artigau $^{a, d}$, Frank Havermeyer ${ }^{b}$, Keith Matthews $^{c}$, \\ Christophe Moser $^{b}$, Demetri Psaltis ${ }^{c}$, Gregory J. Steckman ${ }^{b}$ \\ ${ }^{a}$ Photon etc. inc., 432 boul. des Prairies, Laval, Canada \\ ${ }^{b}$ Ondax inc. 850 E. Duarte Road, Monrovia, CA, USA \\ ${ }^{c}$ California Institute of Technology, 1200 E. California blvd., Pasadena, CA, USA \\ ${ }^{d}$ Université de Montréal, C.P. 6128, Succ. centre-ville, Montréal, Québec, Canada
}

\begin{abstract}
Near-infrared emission from atmospheric $\mathrm{OH}$ radicals is known to severely affect astronomical observations. Until now, only complex dispersive instruments were partially capable of removing this unwanted background, which is composed of hundreds of narrow emission lines. Recent development in photosensitive glass and holographic recording technologies now allow the elaboration of filters with a large number of narrow reflecting bands well matched to $\mathrm{OH}$ lines. This technology shows promise for removing many tens of lines in the $J, H$, and $K$ bands. That would result in a many fold increase in imaging and low resolution signal-to-noise ratio. Filters with 10 lines have been tested and show the appealing possibilities of these new devices.
\end{abstract}

Keywords: astronomy, filter, near infrared, oh, atmospheric, telluric, lines suppression

\section{INTRODUCTION}

Near infrared wavelengths (1 to 2.5 micrometers) are increasingly seen as the best window to observe a wide range of fascinating astronomical phenomena, from the nascent universe to the birth of stars, to direct observations of exo-planets. These wavelengths are indeed less prone to interstellar extinction, and are easier to correct for atmospheric perturbations than their visible counterpart. However, NIR observations greatly suffer from night sky emission mainly caused by excited $\mathrm{OH}$ radicals in the atmosphere. These narrow emission lines dominates the inter-line sky emission by a many orders of magnitude. Removing a few tens of these lines can lead to a twofold or threefold increase in signal-to-noise ratio (SNR). When keeping in mind that SNR is proportional to the telescope diameter, such filters could procure a SNR gain equivalent to a $30 \mathrm{~m}$ class telescope when used on a 8-10 m telescope.

Many attempts to remove this background emission have been made over the last decades. They either rely on expensive dispersion/recombination techniques ${ }^{1}$ or on very thick multilayer interference filters. ${ }^{2}$ None of these techniques is capable of efficiently filtering the narrow airglow lines without severely affecting the astronomical signal. On the other hand, the projected ability of thick holographic filters to remove numerous lines with a spectral width of less than $0.2-0.3 \mathrm{~nm}$ with low transmission loss is unprecedented and will lead to a major evolution in infrared imaging and low resolution spectroscopy.

\section{FILTER DESIGN}

To understand the unique value of holographic technology for the fabrication of multi-narrow band filters, it helps to compare it with the current state of the art filtering technology of thin film interference filters.

The thin film filters available commercially are limited in thickness to a couple of tens of micrometers. Single band filters with up to one hundred layers have been fabricated for the telecommunication industry. The FWHM of these filters has reached $0.2 \mathrm{~nm} .{ }^{3}$ The deposition of the layers has to be well controlled for every layer. Today, $120 \mathrm{~mm}$ wafers with 100 layers have good homogeneity only on $7 \%$ of the surface. Due to the limitation on the

Send correspondence to sbo@photonetc.com 


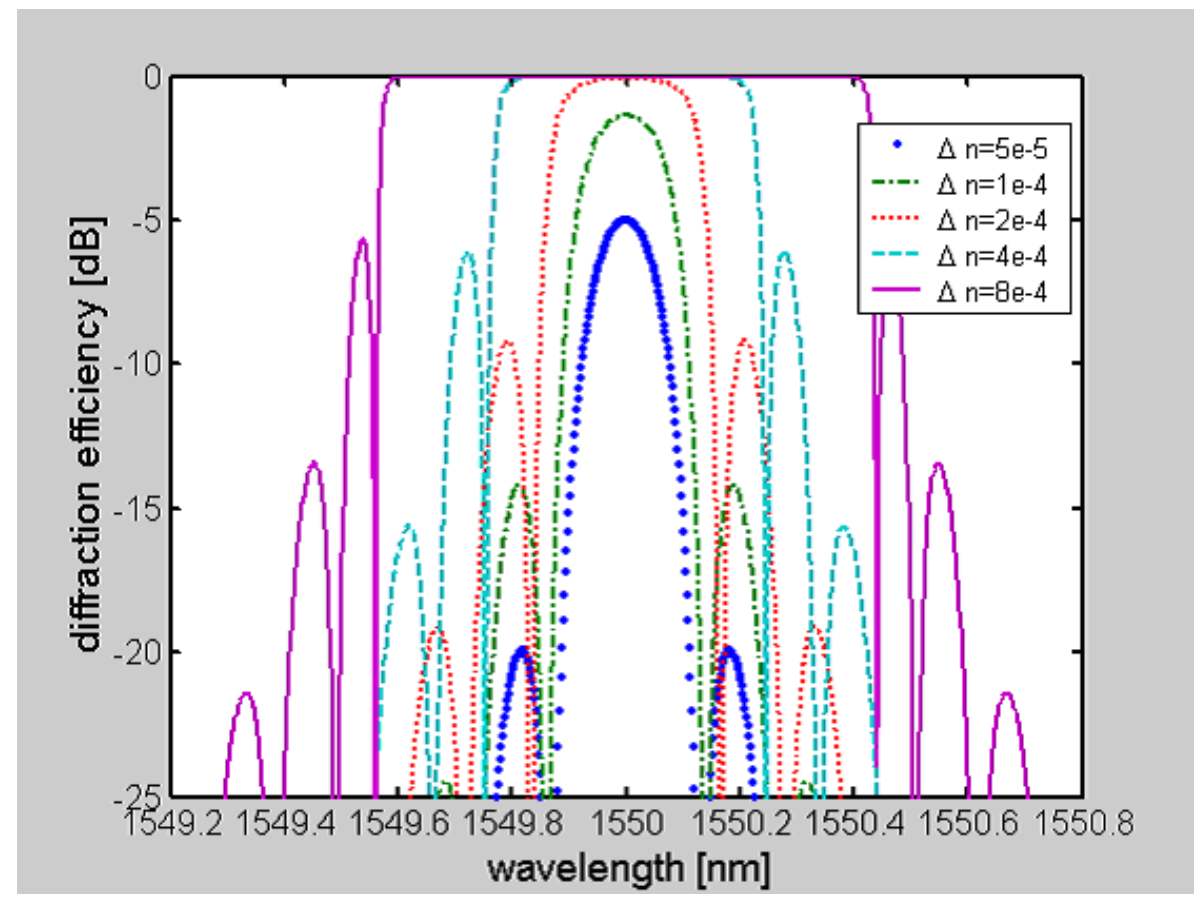

Figure 1. Dependence of spectral bandwidth on refractive index modulation amplitude for strong volume gratings. The apodized gratings are recorded in a $10 \mathrm{~mm}$ thick photosensitive glass $(\mathrm{n}=1.486)$.

number of layers, the type of filter profiles required to suppress narrow band lines of $\mathrm{OH}$ radicals is simply not achievable. Rugate filters, which use a sinusoidal variation of the index rather than a step index, have been shown theoretically to provide three passbands of width $25 \mathrm{~nm} .{ }^{2}$ For an effective suppression of the $\mathrm{OH}$ lines, at least 10 bands of width $0.2 \mathrm{~nm}$ are required. This requirement places a tough challenge for the state of the art technology.

In contrast, holographic technology inherently provides many thousands of layers that enable the design of complex filter profiles that is not possible with thin film deposition. Holographic recording in photosensitive glass material of several millimeter thickness provides a technology platform for controlling the filter profile with better than $0.1 \mathrm{~nm}$ spectral features and ninety percent efficiency. The recent advances in glass material with low absorption and high index contrast at Ondax are enabling the fabrication of these complex filters.

A volume hologram is a three-dimensional (3D) grating created by recording the interference pattern of two mutually coherent light beams, the reference and signal, in a volume medium. The 3D nature of volume holograms offers some desirable properties such as high diffraction efficiency (close to 100\%), high wavelength selectivity and the ability to multiplex multiple holograms in the same volume. Two different theories are generally used to study the diffraction of light from volume holograms. In the weak grating regime, which means that higher-order scattering by the hologram is negligible, the Born's approximation is often used to predict the diffraction efficiency of a volume hologram. However, in the strong grating regime, the Born's approximation breaks down and coupled wave theory ${ }^{4}$ must be applied.

The bandwidth of highly diffracting holograms is proportional to the refractive index change:

$$
\Delta \lambda=\lambda_{0} \frac{\Delta_{n}}{2 n}
$$

$\Delta \mathrm{n}$ in turn is determined by the recording exposure time. Therefore, the spectral bandwidth of each hologram can be controlled by carefully choosing the exposure time during the hologram recording. The diffraction efficiencies of five reflection gratings with different index modulation depths are plotted in Figure 1. It can be 


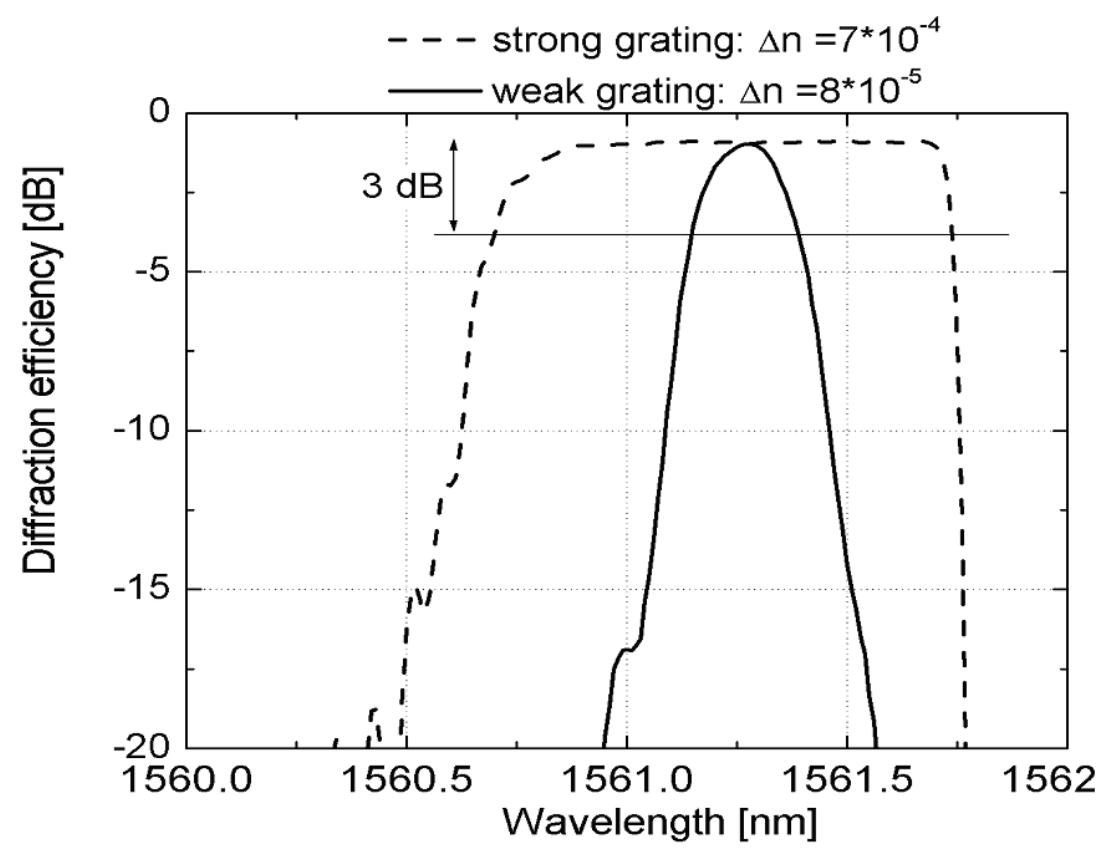

Figure 2. Experimental measurement of the spectral response of two reflection gratings recorded in a $14 \mathrm{~mm}$ thick glass material.
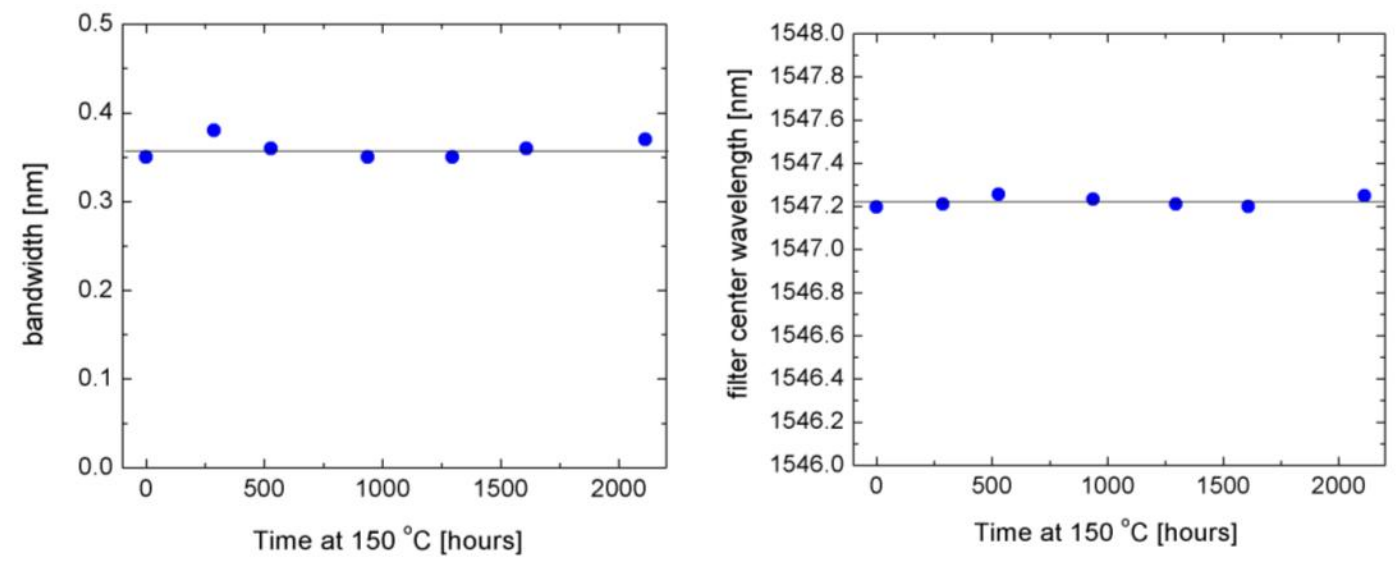

Figure 3. Stability of the central filter frequency and bandwidth of the holographic filter at 150 degree Celsius.

seen that the spectral bandwidth starts to increase as $\Delta \mathrm{n}$ reaches $5 \times 10^{-5}$. When $\Delta \mathrm{n}$ is below $5 \times 10^{-5}$, the grating is still in the weak grating regime, and the spectral bandwidth is determined by the grating length. Figure 2 shows the experimental result of two reflection gratings recorded in a glass material. This result demonstrates that the bandwidth of single filters can be manipulated by adjusting the exposure time.

Another inherent advantage of holographic filters are their relative robustness. No fragile coating prevent polishing and regular cleaning. To test their lifetime, the filter in Figure 2 was submitted to prolonged exposure to elevated temperature. The filter amplitude has shown no degradation after more than 2000 hours at 150 degrees Celsius. The filter quality is measured by its center frequency stability and bandwidth. The result of 


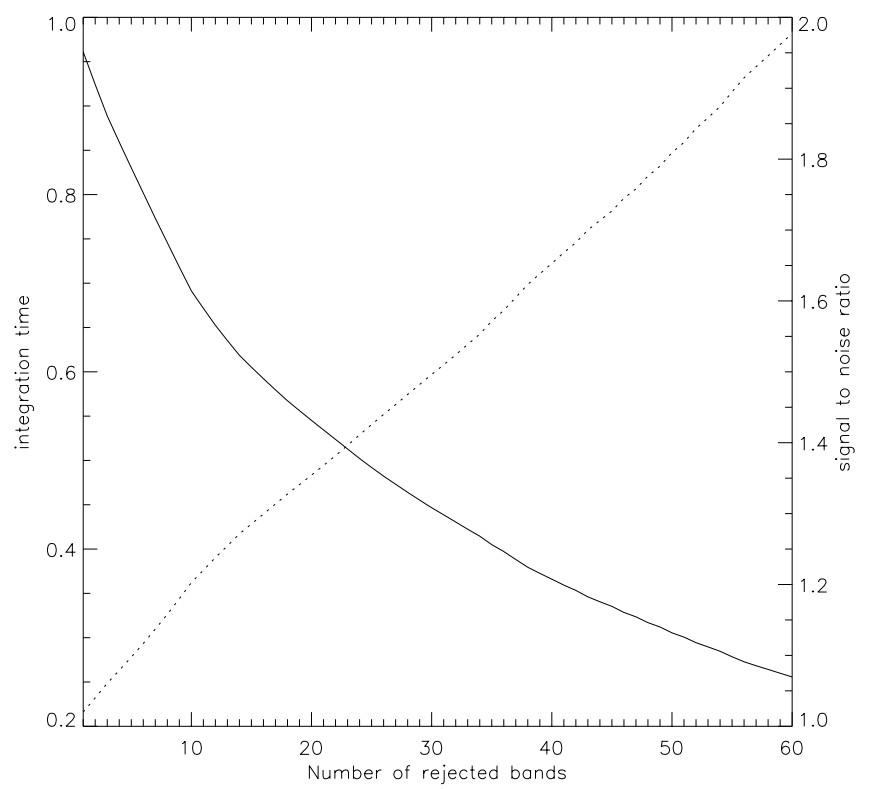

Figure 4. Simulation of the gain in integration time and signal to noise when using a multi-notch filter in a f/16 beam.

this accelerated aging test at elevated temperatures in shown in figure 3.

\section{EXPECTED PERFORMANCE}

The center wavelength of the holographic filter depends on the incidence angle inside the material according to the relation : $\lambda_{R}=\Lambda n \sqrt{2(1+\cos (2 \alpha))}$, where $\Lambda$ is the grating period and $\mathrm{n}$ the index of refraction. The bandwidth of the filter needs to be large enough to accommodate for the wavelength shift caused by the off-axis angles in the imaging system of the telescope. With a focal ratio of 16 , the shift is equal to $0.3 \mathrm{~nm}$ at $1.5 \mu \mathrm{m}$.

A more rigorous analysis of the filtering response of a telescope beam with a given F-number by the holographic filter has been developed. An IDL* simulation evaluates the signal-to-noise ratio of infrared imaging of an extended source in the astronomical $J(1-1.25 \mu \mathrm{m}), H(1.5-1.8 \mu \mathrm{m})$, and $K(2-2.5 \mu \mathrm{m})$ band. It takes into account a real infrared sky spectrum, including OH-lines and thermal emission, a multi-notch filter with various number of reflecting bands, bandwidths, as well as reflection and out-of-band efficiencies. It also includes, detector read-out noise and dark current. The simulation shows the possibility of increasing observing efficiency by large factors. For example, one can half the observing time for full $\mathrm{H}$ band imaging by removing $25 \mathrm{OH}$ lines. Figure 4 shows the gain in integration time and in signal-to-noise against the number of $\mathrm{OH}$ emission line removed. As a more visual example, Figure 5 shows simulated observations of the Infrared Hubble Deep Field with a $10 \mathrm{~m}$ telescope, with and without a 20 line filter compare to HST images.

The gain is even more dramatic when restricting the observations to a sub-band. Indeed, the challenge in building holographic filters reside more in the number of reflecting bands than in the band density. It is thus interesting to explore the possibilities for narrower band than $J, H$, and $K$. As an example, Figure 6 shows that removing 10 lines from the still fairly large 1.49-1.6 $\mu \mathrm{m}$ band reduces the observing time by half to reach a given signal-to-noise.

\footnotetext{
*Research Systems: www.rsinc.com
} 

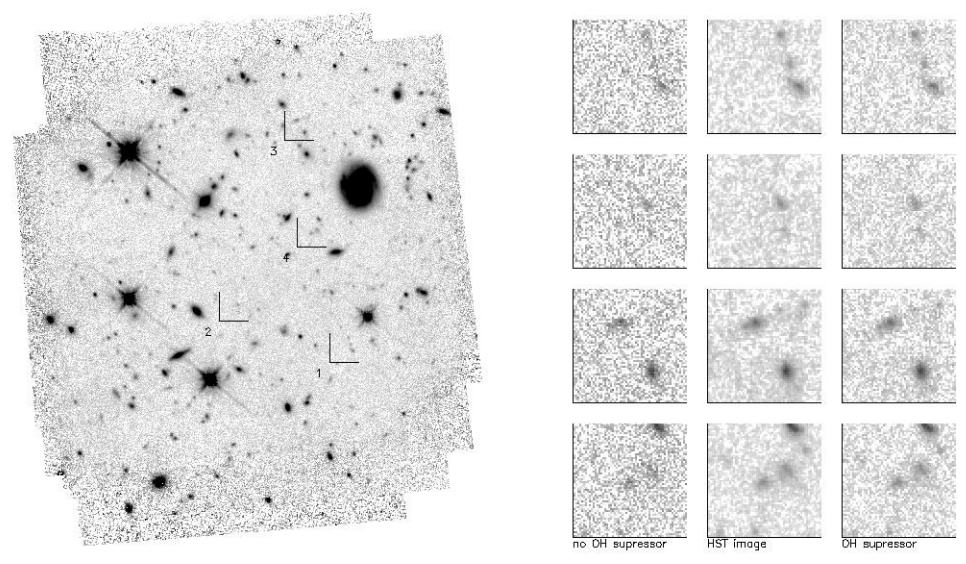

Figure 5. Simulations of ground based observation of the Hubble deep field with a $10 \mathrm{~m}$ telescope, with and without multi-notch filter compared to HST images. Angular resolution has been kept the same.
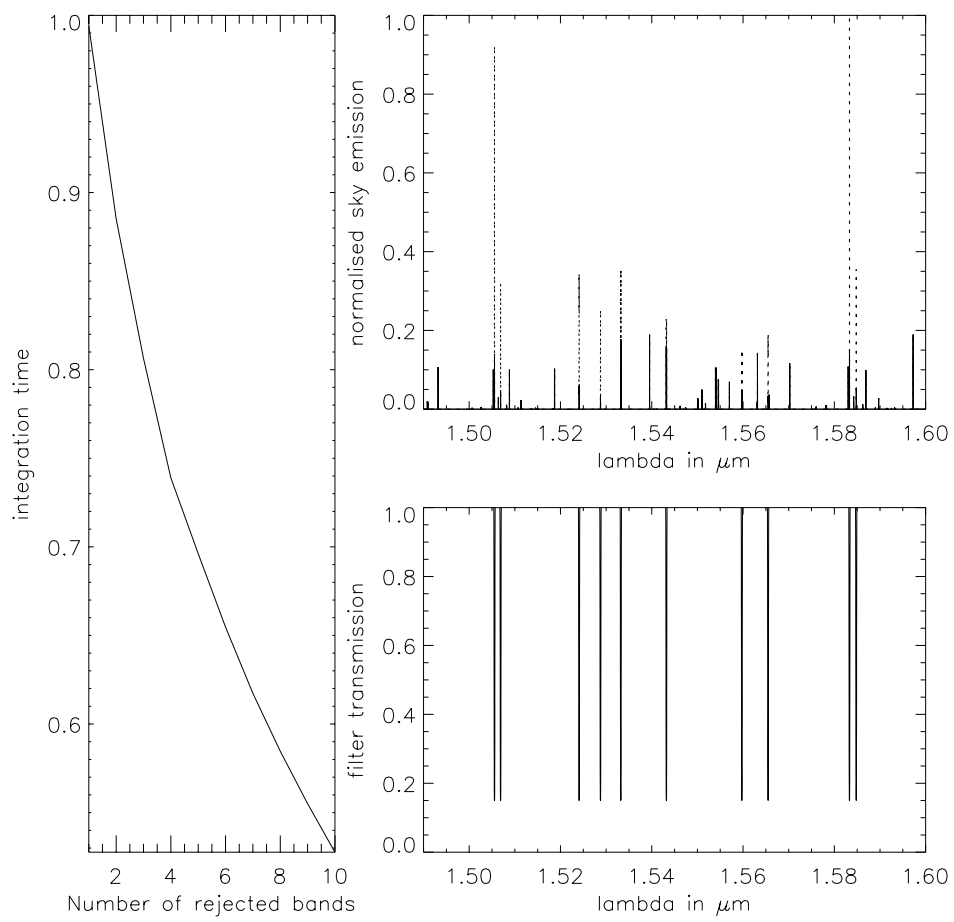

Figure 6. Simulation of the gain in integration time and signal to noise when using a 10 notch filter in a f/16 beam. 


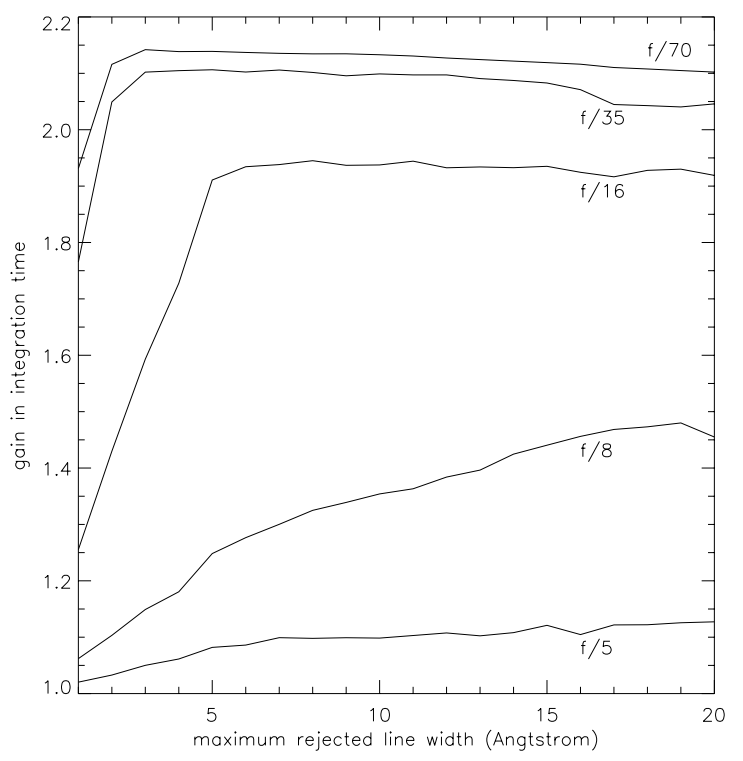

Figure 7. Efficiencies of a 20 line filter for the $1.45-1.6 \mu \mathrm{m}$ band against the notches maximum width for various $\mathrm{f}$ numbers. From top to bottom: f/70, f/35, f/16, f/8, f/5.

Because of the wavelength shift due to off-axis angle, filter efficiency is a function of notch bandwidth. As shown in Figure 7, a steady efficiency increase is seen for increasing bandwidth and reaches a maximum value that depends on the F number, as expected.

Increasing bandwidth is thus desirable but requires a high modulation of the glass index of refraction. Current technology allows up to $0.2 \mathrm{~nm}$ bandwidth but larger bandwidth are expected to be available soon.

\section{PRELIMINARY RESULTS}

Several experimental filters have already been produced to demonstrate the applicability of the technique for the production of a filter to simultaneously reject multiple infrared $\mathrm{OH}$ sky emission lines. Figure 8 shows a filter with 10 reflection narrow bands $(F W H M=0.1 \mathrm{~nm})$. The filter was recorded 5 times at different place on the same substrate. All 5 sets of 10 lines fell at the desired wavelength at ${ }_{-0.08 \mathrm{~nm}}^{+0.02 \mathrm{~mm}}$.

Current recording technology limits the physical width off the filter to a few millimeters. Their length on the other hand is not intrinsically limited. It is however possible to bond together many filter slices to build large format filters suitable for today's telescope. Figure 9 shows a $18 \times 30 \mathrm{~mm}$ assembly of $3 \times 30 \mathrm{~mm}$ slices. In this configuration, the slice interface need to be blacken to avoid stray light due to total internal reflection. These losses are expected to be greatly reduced in the near future by increasing the slice width to $9 \mathrm{~mm}$.

\section{CONCLUSION}

Multi-notch volume holographic filters under developments show promise of substantially increasing efficiency of infrared imaging and low resolution spectroscopy. They have large reflection efficiencies and narrow bandwidths, well adapted for atmospheric $\mathrm{OH}$ emission lines filtering. Filter with up to 10 lines are currently produced and their use in near-infrared can reduce the integration time by a large amount. For example, a given signal-to-noise can be reached in half the time for a $110 \mathrm{~nm}$ window in $H$ band.

Improving the filter performances present important technological challenges. One would like to increase the number of lines and line width. Both require a improvement in the glass technology which is indeed in constant evolution. New recording techniques are also explored that can improve the number of lines as well as the size 


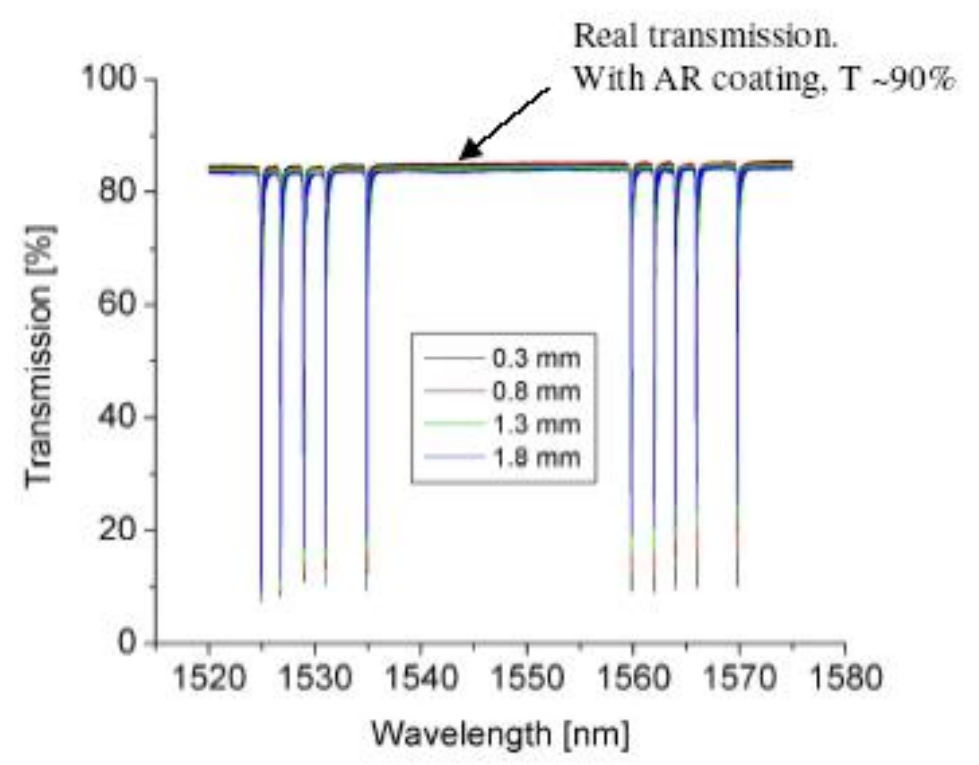

Figure 8. Transmission spectrum of a 10 line filter.

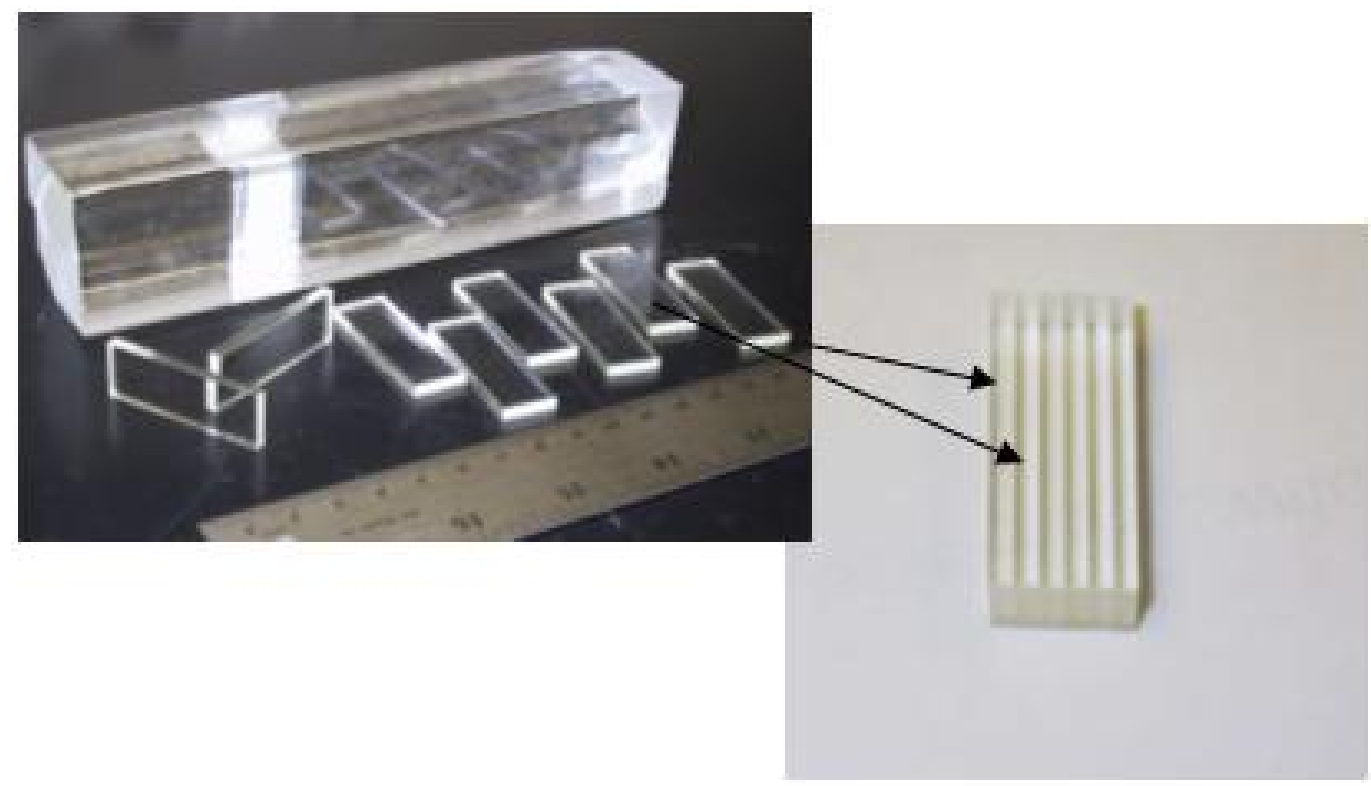

Figure 9. Mosaic of filter slices. 
of the sample. None of these limitation are intrinsic to the concept and it is foreseeable that large format filters of more than 20 lines, adapted to fast beams can be built in a very few years.

\section{ACKNOWLEDGMENTS}

This material is based in part upon work supported by the National Science Foundation under Grant No. 0338906

\section{REFERENCES}

1. R. Content and J. R. P. Angel, "Design and model demonstration of a camera that removes the infrared $\mathrm{OH}$ background from two-dimensional images," in Proc. SPIE Vol. 2198, p. 757-762, Instrumentation in Astronomy VIII, David L. Crawford; Eric R. Craine; Eds., pp. 757-762, June 1994.

2. A. R. Offer and J. Bland-Hawthorn, "Rugate filters for OH-suppressed imaging at near-infrared wavelengths," MNRAS 299, pp. 176-188, Aug. 1998.

3. M. Scobey. OFC2002, 2002.

4. H. Kogelnik, "Coupled wave theory for thick hologram gratings," Bell System Tech. J. 48(9), p. 2909, 1969. 